\title{
Search for scalar top quark pair production in the top corridor region at the CMS experiment
}

\section{Andrea Trapote Fernández* on behalf of the CMS Collaboration}

Universidad de Oviedo, Instituto Universitario de Ciencias y Tecnologías Espaciales de Asturias (ICTEA) Oviedo, Spain

E-mail: andrea.trapote.fernandez@cern.ch

A search for scalar top quark pair production at the LHC with the CMS experiment is presented. This search targets a region of parameter space where the kinematics of top squark pair production and top quark pair production are very similar because of the mass difference between the top squark and the neutralino being close to the top quark mass. The search is performed with the full Run 2 data set of proton-proton collisions at a centre-of-mass energy of $13 \mathrm{TeV}$, collected by the CMS detector, using events containing dilepton pairs with opposite charge. A DNN algorithm is used to separate signal from background.

9th Edition of the Large Hadron Collider Physics Conference - LHCP2021

July 7 - 12, 2021

Paris, (virtual meeting)

${ }^{*}$ Speaker 


\section{Contents}

1 Introduction 2

2 Top corridor region search 2

3 Summary 3

\section{Introduction}

Supersymmetry (SUSY) is a promising extension of the Standard Model (SM) that introduces a relation between bosons and fermions and resolves some problems that the SM cannot explain, for example, the hierarchy problem. It also provides a dark matter candidate.

Different simplified models exist that describe the production of top squark ( $\mathfrak{t})$ pairs, but this poster focuses on the so-called "T2tt" model from the Simplified Model Spectra (SMS) [1], in which a $100 \%$ branching ratio is assumed for the top squark to top quark + lightest neutralino $\left(\tilde{\chi}_{1}^{0}\right)$ decay. This poster presents the combination[6] of previously published searches for the pair production of SUSY top quark partners in final states without leptons [3], with one [4], or with two charged leptons [5], in events from proton-proton (pp) collisions at a center-of-mass energy of $13 \mathrm{TeV}$ at the CERN LHC, corresponding to an integrated luminosity of $137 \mathrm{fb}^{-1}$ collected by the CMS experiment. It also includes a new analysis [6] targeting a parameter space where the mass difference between the top squark and the neutralino is close to the top quark mass. In this region of the parameter space, the signal has very similar kinematics to the top quark pair production (tit) background, and it is very difficult to separate them. This region is referred to as "top corridor" and to improve the separation a Deep Neural Network (DNN) is used.

\section{Top corridor region search}

The top corridor region corresponds to events where the mass difference between $\tilde{\mathrm{t}}$ and $\tilde{\chi}_{0}$ is close to the top mass, in which signal and t $\bar{t}$ background have similar kinematics, especially at low $\tilde{\chi}_{0}$ masses. Therefore, signal events can only be detected as an excess on the tit cross section and it is important to have an accurate estimation of the background to be sensitive to such SUSY signals.

The signal model used was the direct pair production of top squarks, for a sequence of values of the top squark mass in the range $145-295 \mathrm{GeV}$ and a mass difference between $\tilde{\mathrm{t}}$ and $\tilde{\chi}_{0}$ from 0 to $30 \mathrm{GeV}$.

This analysis uses the full Run 2 data set and events are selected if they contain an opposite-sign lepton pair (electron or muons). Then, to suppress Drell-Yan and other boson backgrounds, also are required at least two jets and at least one b-tagged jet. This set of requirements is referred to as the baseline selection. 
After the baseline selection, most of the background events (about 98\%) are expected to come from $t \bar{t}$. To suppress this background, the signal region is defined with the requirements $p_{T}^{\text {miss }}>50$ $\mathrm{GeV}$ and $m_{T 2}(\ell \ell)>80 \mathrm{GeV}$.

One of the main discriminating variables between signal and $t \bar{t}$ is $m_{T 2}(\ell \ell)$ [2], which has an endpoint for $\mathrm{t} \overline{\mathrm{t}}$ at the $\mathrm{W}$ boson mass, while signal events are expected to populate the tails of the distribution. Higher discrepancies between signal and $t \bar{t}$ appear at large values of the $m_{T 2}(\ell \ell)$ distribution, and they are larger at high $\tilde{\chi}_{0}$ mass. The $m_{T 2}(\ell \ell)$ observable is computed as:

$$
m_{T 2}(\ell \ell)=\min _{\vec{p}_{\mathrm{T}, 1}^{\text {miss }}+\vec{p}_{\mathrm{T}, 2}^{\text {miss }}=\vec{p}_{\mathrm{T}}^{\text {miss }}}\left(\max \left[m_{T}\left(\vec{p}_{\mathrm{T}}^{\ell 1}, \vec{p}_{\mathrm{T}, 1}^{\text {miss }}\right), m_{T}\left(\vec{p}_{\mathrm{T}}^{\ell 2}, \vec{p}_{\mathrm{T}, 2}^{\text {miss }}\right)\right]\right),
$$

where $m_{T}$ is the transverse mass, and $\vec{p}_{\mathrm{T} 1}^{\text {miss }}$ and $\vec{p}_{\mathrm{T} 2}^{\text {miss }}$ correspond to the estimated transverse momenta of two neutrinos that are presumed to determine the total $\vec{p}_{\mathrm{T}}^{\text {miss }}$ of the event. The transverse mass is calculated for each lepton-neutrino pair, for different assumptions of the neutrino $p_{T}$.

In order to maximize the sensitivity and to exploit all the differences between the signal and $t \bar{t}$ background, a multivariate analysis is implemented using a parametric DNN [7]. The training was done using events passing the baseline selection in order to use the separation power of different observables over a large range. A total of 13 variables are selected for the training, including the $\tilde{\mathrm{t}}$ and $\tilde{\chi}_{0}$ masses in order to exploit the kinematic differences to maximize sensitivity. For background events those values are randomly taken, to avoid introducing correlations. In this way, a specific model for each signal point training a single DNN is achieved.

The data and predicted distributions for the DNN response in the signal region are combined in the nine channels ( 3 data-taking period $\mathrm{x} 3$ lepton flavor combinations of the two leading leptons) in order to maximize the sensitivity to the signal. Each of the distributions is computed for different values of the mass parameters and compared to the prediction for the signal model with the corresponding masses.

The exclusion limits on the production cross section of top squark pairs at $95 \%$ confidence level (CL) are calculated and the full top corridor region is excluded for the first time, shown in Figure 1.

\section{Summary}

This poster focuses on the dedicated search of top squark pair production in the 'top corridor' region, where other analyses do not have enough sensitivity and the full region, ranging top squark masses of $145-295 \mathrm{GeV}$ and a mass difference between $\tilde{\mathrm{t}}$ and $\tilde{\chi}_{0}$ from 0 to $30 \mathrm{GeV}$, is excluded at $95 \%$ CL. It also summarizes the combination of the last exclusion limits achieved by the CMS Collaboration with the full Run 2 data set for top squark pair production on a simplified SUSY model assuming a $100 \%$ branching fraction to a top quark and a neutralino, excluding top squarks with masses up to $1.3 \mathrm{TeV}$. 


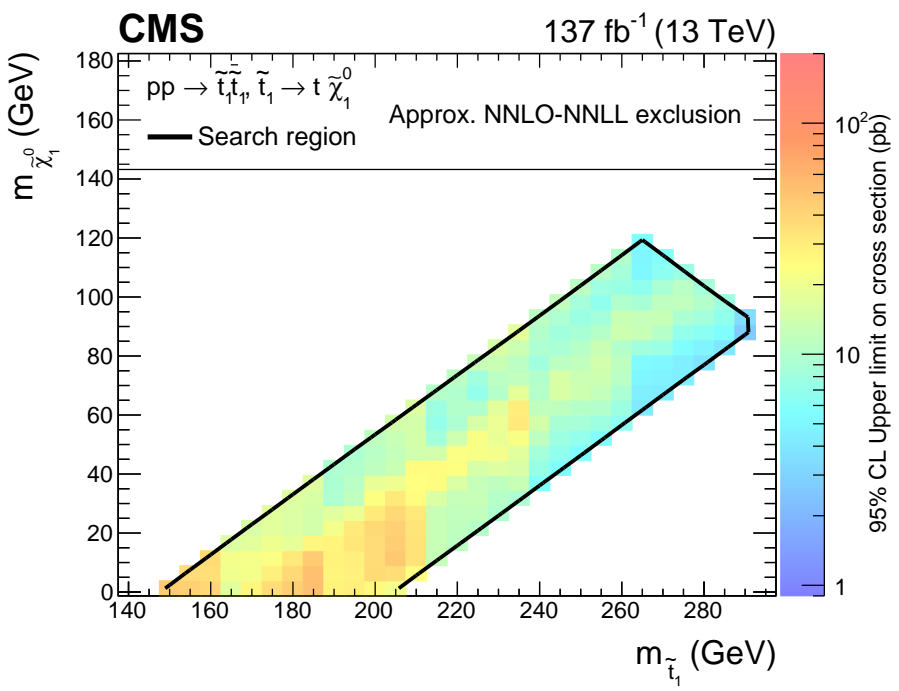

Figure 1: Upper limit at $95 \% \mathrm{CL}$ on the signal cross section as a function of the top squark and neutralino masses in the top quark corridor region [6]. The model is excluded for all of the colored region inside the black boundary.

\section{References}

[1] Alwall, Johan and Schuster, Philip C. and Toro, Natalia, Simplified models for a first characterization of new physics at the LHC, Phys. Rev. D 79 (2009) 38. doi:10.1103/PhysRevD.79.075020

[2] C.G Lester and D.J Summers, Measuring masses of semi-invisibly decaying particle pairs produced at hadron colliders, Physics Letters B 463 (199) 99. doi:10.1016/S0370-2693(99)009454

[3] CMS Collaboration, Search for top squark production in fully-hadronic final states in protonproton collisions at 13 TeV, Phys. Rev. D. 104 (2021) 46. doi:10.1103/PhysRevD.104.052001

[4] CMS Collaboration, Search for direct top squark pair production in events with one lepton, jets, and missing transverse momentum at $13 \mathrm{TeV}$ with the CMS experiment JHEP 05 (2020) 032. doi:10.1007/JHEP05(2020)032

[5] CMS Collaboration, Search for top squark pair production using dilepton final states in $p p$ collision data collected at 13 TeV, Eur. Phys. J. C 81 (2021) 3. doi:10.1140/epjc/s10052-02008701-5

[6] CMS Collaboration, Combined searches for the production of supersymmetric top quark partners in proton-proton collisions at $13 \mathrm{TeV}$, Eur. Phys. J. C arXiv:2107.10892

[7] Baldi, P., Cranmer, K., Faucett, T. et al., Parameterized neural networks for high-energy physics, Eur. Phys. J. C 76, 235 (2016). doi:10.1140/epjc/s10052-016-4099-4

CMS Collaboration, Combined searches for the production of supersymmetric top quark partners in proton-proton collisions at $13 \mathrm{TeV}$, Eur. Phys. J. C arXiv:2107.10892 